Boise State University

ScholarWorks

8-1-2007

Instantaneous Spectral Analysis: Time-Frequency Mapping via Wavelet Matching with Application to Contaminated-Site Characterization by 3D GPR

John H. Bradford

Boise State University

Yafei Wu

Anadarko Petroleum Corporation 


\title{
Instantaneous spectral analysis: Time-frequency mapping via wavelet matching with application to contaminated-site characterization by 3D GPR
}

\author{
JoHn H. BRADFORD, Boise State University, Boise, USA \\ YAFEI WU, Anadarko Petroleum Corporation, Houston, USA
}

Spectral decomposition, by which a time series is transformed from the $1 \mathrm{D}$ time/amplitude domain to the $2 \mathrm{D}$ time/spectrum domain, has become a popular and useful tool in seismic exploration for hydrocarbons. The windowed, or short-time Fourier transform (STFT) was one early approach to computing the time-frequency $(t-f)$ distribution. This method relies on the user selecting a fixed time window, then computing the Fourier spectrum within the time window while sliding the window along the length of the trace. The primary limitation of the STFT is the fixed window which prevents either time localization of high frequency components (if a long window is used) or spectral resolution of the low-frequency components (if a short window is used).

The Wigner Distribution (WD) is another approach to computing the $t$ - $f$ distribution (Boudreaux-Bartels, 1985). The WD, which is the Fourier transform of the instantaneous autocorrelation, has time and frequency localization, but suffers from cross-term interference. More recently, waveletbased approaches have become popular because of superior time resolution while maintaining good spectral resolution. The continuous wavelet transform (CWT) does not provide a direct measure of the $t-f$ distribution, but rather maps the data into the time-scale domain. Scale is a measure of frequency band and while related to the frequency content of the data, it is not a direct measure of the frequency spectrum.

Sinha et al. (2005) describe an approach to computing the $t$ - $f$ distribution directly from the CWT. Here, we present an alternative wavelet-based approach to computing the $t$ $f$ distribution which we describe as a wavelet-matching decomposition (WMD). We originally developed this method for applications in seismic and ground-penetrating radar (GPR) attenuation analysis (Bradford, 1999; Bradford and $\mathrm{Wu}$, 1997) while at the Houston Advanced Research Center in the mid-1990s. The WMD is similar to the matching pursuit algorithm derived by Mallat and Zhang (1992), and later applied to seismic data by Chakraborty and Okaya (1995). A number of recent implementations are based on our earlier development of the wavelet-matching algorithm and time-frequency mapping method (e.g. Castagna et al., 2003, and Liu et al., 2004). Although the potential benefits for imaging small-scale features with GPR data are analogous to seismic exploration, application of spectral decomposition in GPR imaging remains limited. One particularly important application is contaminated-site characterization.

Nonpolar organic liquids are collectively referred to as as nonaqueous phase liquids (NAPL) because of their low solubility in water. NAPLs are subcategorized by their density relative to water with DNAPL being denser than water and LNAPL being lighter than water. It follows that DNAPLs tend to sink through the water column until they reach a low-permeability layer, while LNAPLs tend to remain near the water table with free product floating on the water column and residual product present in a smear zone above and below the water table. Both LNAPL and DNAPL leave a zone of residual contamination along their migration routes. Chlorinated solvents (DNAPL) and fuel hydrocarbons (LNAPL) are examples that are commonly found at contaminated sites.

NAPLs typically have low relative permittivity $(\mathrm{K} \sim 2.5)$ and high electric resistivity $\left(\sim 10^{4}-10^{5} \mathrm{ohm}-\mathrm{m}\right)$ relative to water which make them attractive targets for characterization using electric geophysical methods. As the NAPL displaces water in the sediment pore space, a zone of anomalous electric properties may be induced. It has been demonstrated at a number of sites that the simple model of NAPL contamination being associated with low electric conductivity is not always applicable. However, it is also clear that GPR is often sensitive to electric properties anomalies associated with NAPL contamination whether or not those anomalies are consistent with an assumed conceptual model. Methods such as reflection tomography and reflection attenuation analysis may be indicators of the long-wavelength contaminant distribution; however, more typically LNAPLs are present in thin layers floating at the water table. These thin layers are often below the conventional $1 / 4$ wavelength resolution of a radar signal capable of penetrating to the target depth. In these cases, AVO or instantaneous attributes may indicate the presence of contaminants.

Here, we present the WMD algorithm for calculating the $t-f$ distribution in which a band-limited GPR signal is decomposed into a linear combination of phase variant, complex Ricker wavelets. The complex spectrum of each wavelet is then mapped to the time-frequency domain using an adaptive windowing function. In a 3D, multioffset GPR, data set acquired over an LNAPL contamination plume at the former Wurtsmith AFB, Michigan, the spectral maps image details of the contaminated interval that are not resolved with other methods such as conventional instantaneous attributes.

The wavelet matching algorithm. We know from wavelet theory that a time series may be decomposed into a summation of individual wavelets under the condition that the wavelet integrates to zero over infinite time (Daubechies, 1991). The particular form of the wavelet is the wavelet basis. The $t-f$ domain is nonunique, and therefore it is best to choose a wavelet basis that is a good representation of the signal of interest. The Ricker wavelet satisfies the integral constraint and is given by

$$
R(t)=2 l e^{-l(t-t i)^{2}}\left[2 l\left(t-t_{i}\right)^{2}-1\right]
$$

where $1=\pi^{2} f_{i}^{2}, f_{i}$ is the peak frequency of the wavelet, and $t_{i}$ is the time shift from $t=0$ to the center of the wavelet. The Ricker wavelet basis is useful in GPR signal analysis because the Ricker wavelet, or a phase variant form, provides a good representation of many commercial GPR signals. We obtain a continuous wavelet decomposition using a wavelet matching algorithm similar to the matching pursuit method developed by Mallat and Zhang (1992). We assume that the signal, $S(t)$, is band-limited, and can be represented by linear summation of wavelet atoms. The signal is then specified by four 


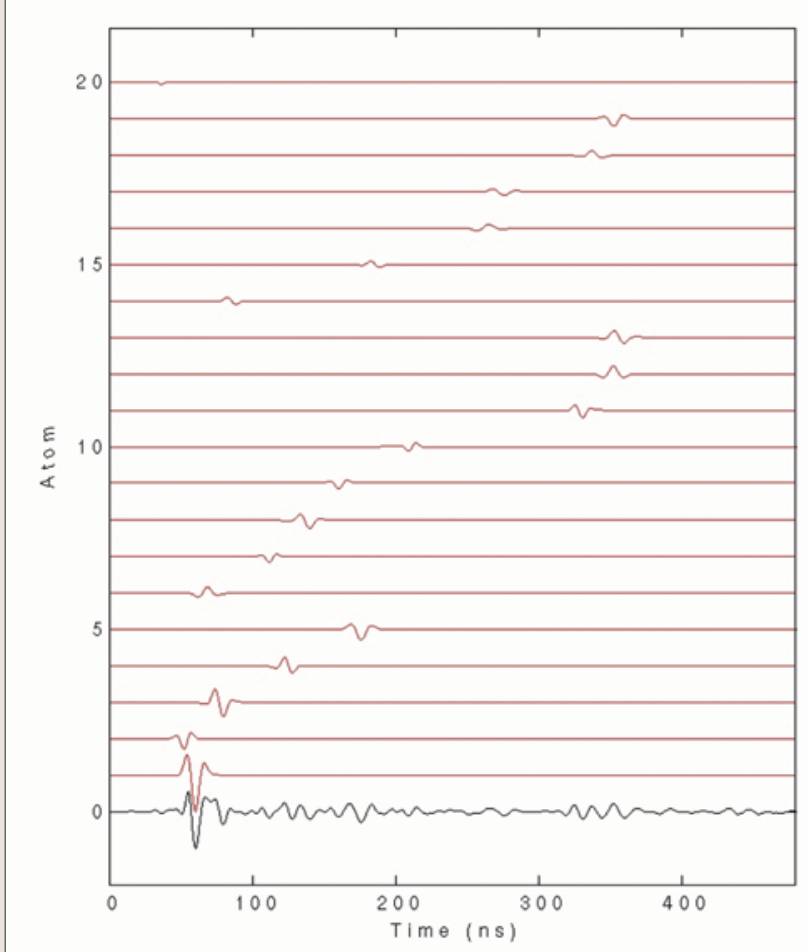

Figure 1. A field GPR trace (black) is plotted along with the first 20 atoms of the decomposition (red).

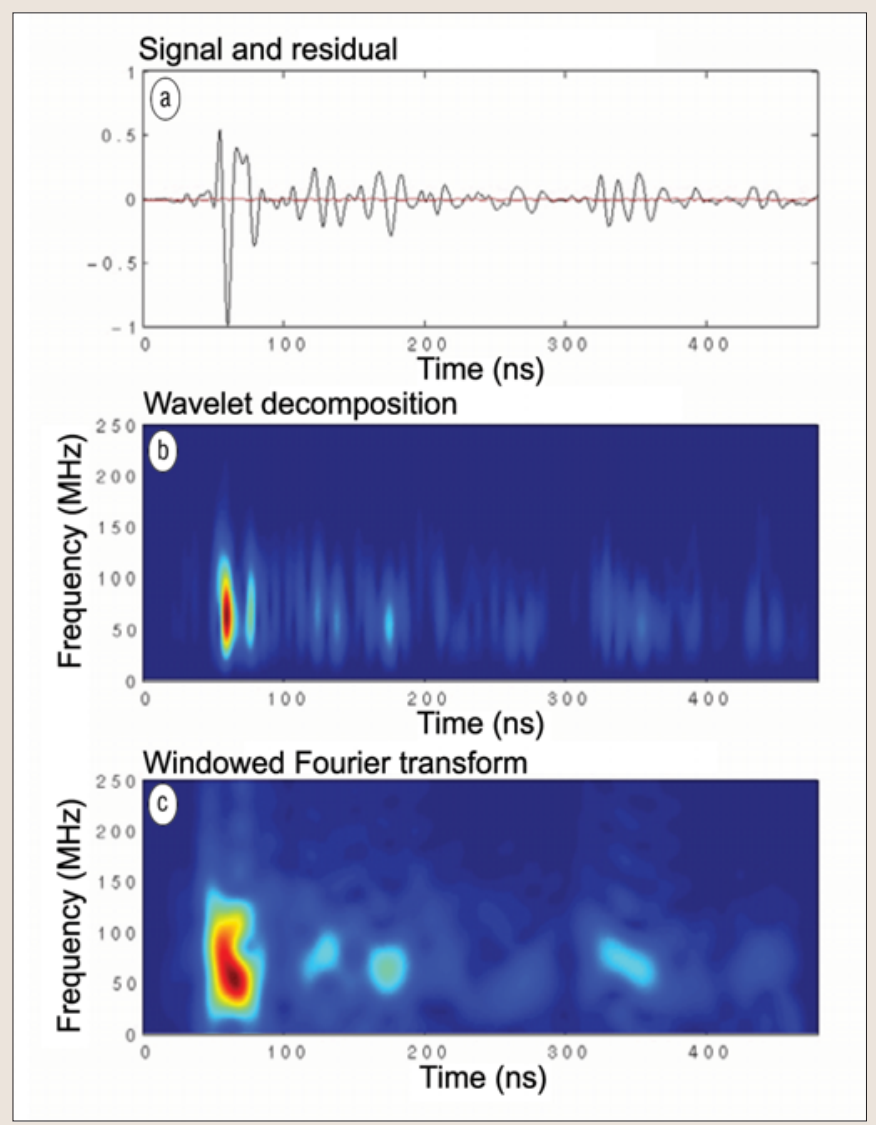

Figure 2. (a) GPR signal reconstructed with 139 wavelet atoms. The residual, shown in red, contains less than 0.001 of the original signal energy. $\mathrm{t}$-f representations via (b) the wavelet matching algorithm (WMD) and (c) the windowed Fourier transform (STFT). The STFT has good spectral resolution but provides relatively poor time resolution. The WMD provides good time and spectral resolution.

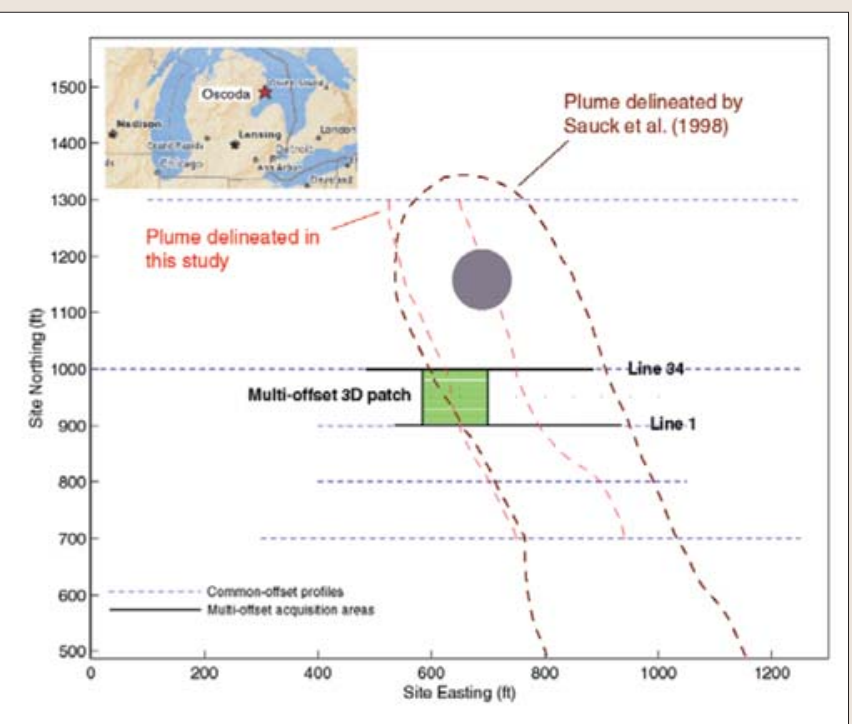

Figure 3. Field site location and survey layout. [Adapted from Bradford (2003)]. Note that distances are in feet, but described as meters in text.

\begin{tabular}{|l|l|}
\hline \multicolumn{2}{|l|}{ Table 1. Acquisition parameters. } \\
\hline $\begin{array}{l}\text { GPR system sensors and } \\
\text { software }\end{array}$ & PE100 w/ $100 \mathrm{MHz}$ \\
\hline Transverse-electric (TE) mode & 34 lines $-0.9 \mathrm{~m}$ line interval \\
\hline Source interval & $0.6 \mathrm{~m}$ \\
\hline Receiver interval & $0.3 \mathrm{~m}$ \\
\hline \# Receivers/source point & 25 \\
\hline TE near-offset & $0.9 \mathrm{~m}$ \\
\hline Recording time & $750 \mathrm{~ns}$ \\
\hline Stacks/shot record & 16 \\
\hline Sampling interval & $0.8 \mathrm{~ns}$ \\
\hline
\end{tabular}

parameters

$$
\mathrm{S}(\omega)=\Sigma R\left(A_{i}, f_{i}, t_{i}, \phi_{i}\right)
$$

where $A_{i}$ is the amplitude. We've also introduced the phase term, $\phi_{i}$, which allows for phase rotation of the wavelet and means that our wavelet basis is not a true Ricker wavelet, which is zero phase, but rather a phase variant form. The amplitude and time of each significant seismic event are estimated by locating the local maxima in the envelope function (absolute value of the complex trace). The phase angle and dominant frequency are then estimated at $t_{i}$ from the phase function and its derivative, respectively (Robertson and Nagomi, 1984). The wavelet atom is then subtracted from the original trace. This process is continued until the energy of the residue is lower than some specified threshold value (Figure 1).

The time extent of the Ricker wavelet is well defined by the Gaussian window function, $G(t)=\exp \left(-l t^{2}\right)$. As we will show later, $G(t)$ also appears in the Wigner distribution. Two dimensional $t-f$ atoms are constructed by mapping the complex Fourier spectra of each Ricker wavelet to the $t-f$ plane using the windowing function $G(t)$. The $t-f$ distribution (Figure 2), WMD(S), is then the linear summation of complex $t-f$ atoms

$$
W M D(S)=\Sigma G_{i}(t) R_{i}(\omega)
$$

where

$$
G_{i}(t) R_{i}(\omega)=K_{i} A_{i} e^{-l_{i}\left(t-t_{i}\right)^{2}} \omega^{2} e^{\frac{-\omega^{2}}{4 l_{i}}} e^{i \omega t_{i}+\phi_{i}}
$$




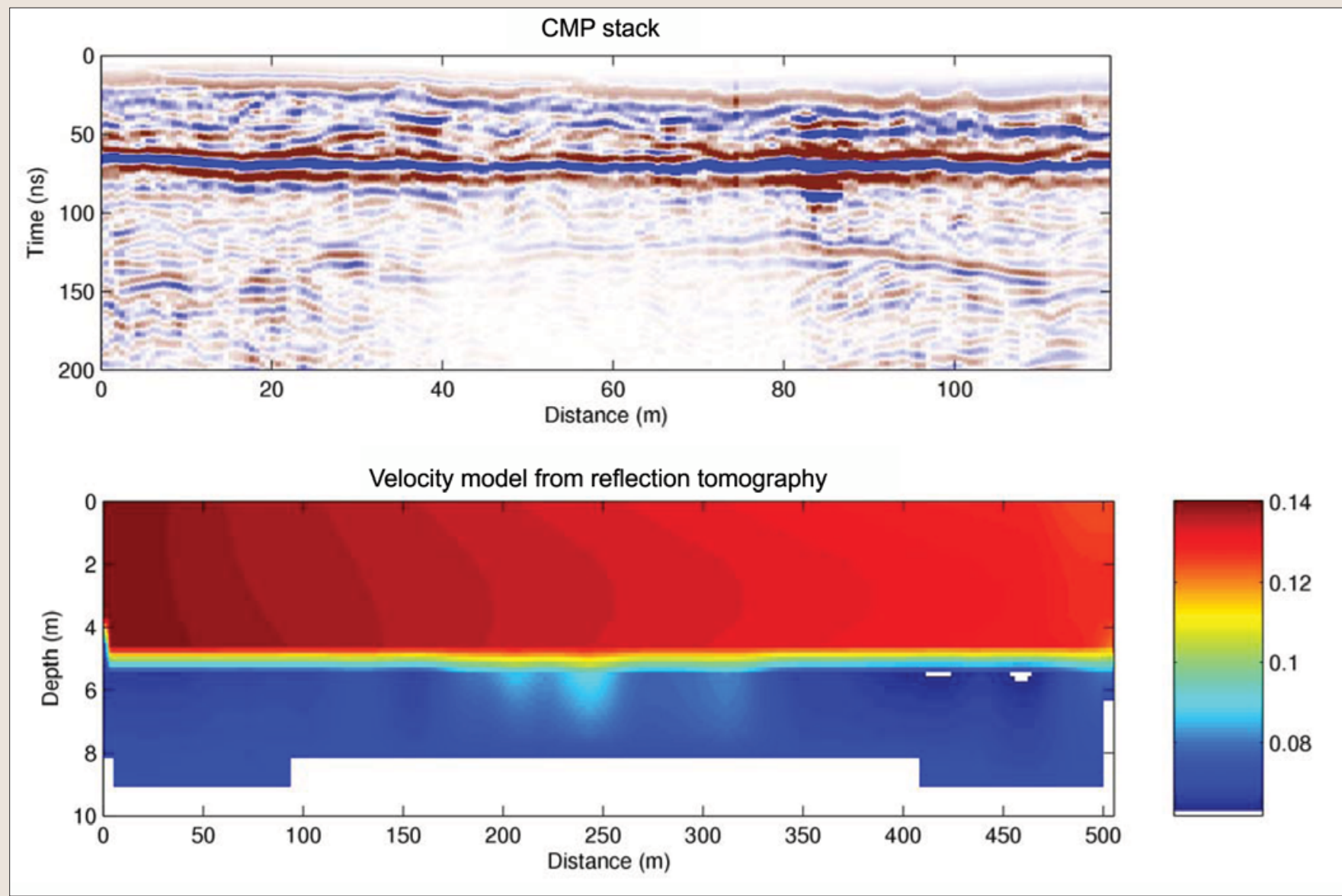

Figure 4. CMP stack along Line 34 (Figure 1), and depth velocity model estimated from reflection tomography. The water table is the prominent reflection at about $60 \mathrm{~ns}$. The plume is evident as a zone of increased attenuation of the radar signal and an increase in radar velocity just below the water table. The velocity anomaly is an indicator of the long-wavelength distribution of contaminates.

and $K$ is a normalization constant.

Since we preserve phase information through the mapping to the $t-f$ plane, the spectra of a multicomponent signal is correctly reconstructed in the $t-f$ domain and our distribution has the following properties

$$
S(\omega)=\int W M D(S) d t
$$

which leads to

$$
\left.S^{2}(\omega)=\left(\int W M D(S) d t\right) *\left(\int W M D(S) d t\right)\right)
$$

where $S^{2}(\omega)$ is the power spectra of the signal $S$ and $S(\omega)$ is the Fourier transform of $S$. It is useful to look at the phase independent form $\left(|\mathrm{WMD}|^{2}\right)$ of our distribution for comparison to the WD, since the WD is always real valued and proportional to the power spectra of the signal. We first consider a single Ricker wavelet, with $t-f$ distribution given by

$$
|W M D|^{2}=K e^{-2 l\left(t-t_{i}\right)^{2}} e^{\frac{-\omega^{2}}{2 l}} \omega^{4}
$$

which is directly proportional to the power spectra. The WD for $R\left(t-t_{i}\right)$ is given by (Bradford, 1999):

$$
\begin{aligned}
& W D(S)=F\left[R\left(t+\frac{\tau}{2}\right) R\left(t-\frac{\tau}{2}\right)\right]= \\
& K_{w} e^{-2 l\left(t-t_{i}\right)^{2}} e^{-\frac{\omega^{2}}{2 l}}\left[\frac{\omega^{4}}{4 l^{2}}+\left(2\left(t-t_{i}\right)^{2}-\frac{1}{2 l}\right) \omega^{2}+4 l^{2}\left(t-t_{i}\right)^{4}-6 l\left(t-t_{i}\right)^{2}+3 / 4\right](8)
\end{aligned}
$$

We see that the WMD is directly proportional to the $\omega^{4}$ term in the WD. As mentioned earlier, we see that the WD contains the window $G_{i}^{2}(t)$. It is interesting to note that the

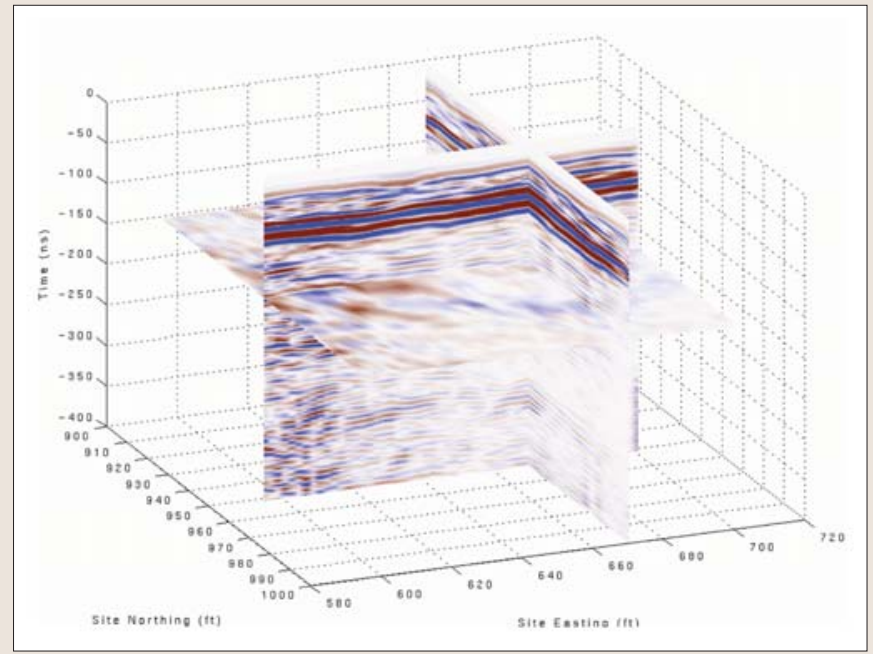

Figure 5. 3D stack volume. See Figure 3 for position relative to plume. The water table at 60 ns is the dominant reflection. Note that distances are in feet but described in meters in text.

WD has a significant dc component $(\mathrm{WD} \neq 0$ at $\omega=0)$. This is surprising since $R(\omega)=0$ at $\omega=0$. The dc component arises from the instantaneous autocorrelation since $R(t)$ is symmetric about $t=t_{i}$. For a number of applications, it is desirable to have a $t-f$ representation that is locally proportional to the power spectra of the data (reflected wavelet). The dc component makes $\mathrm{WD}[(R(t)]$ undesirable. If we take the WD of the complex representation of $R(t)$, we find that the dc terms are suppressed and the WD of the analytic signal 

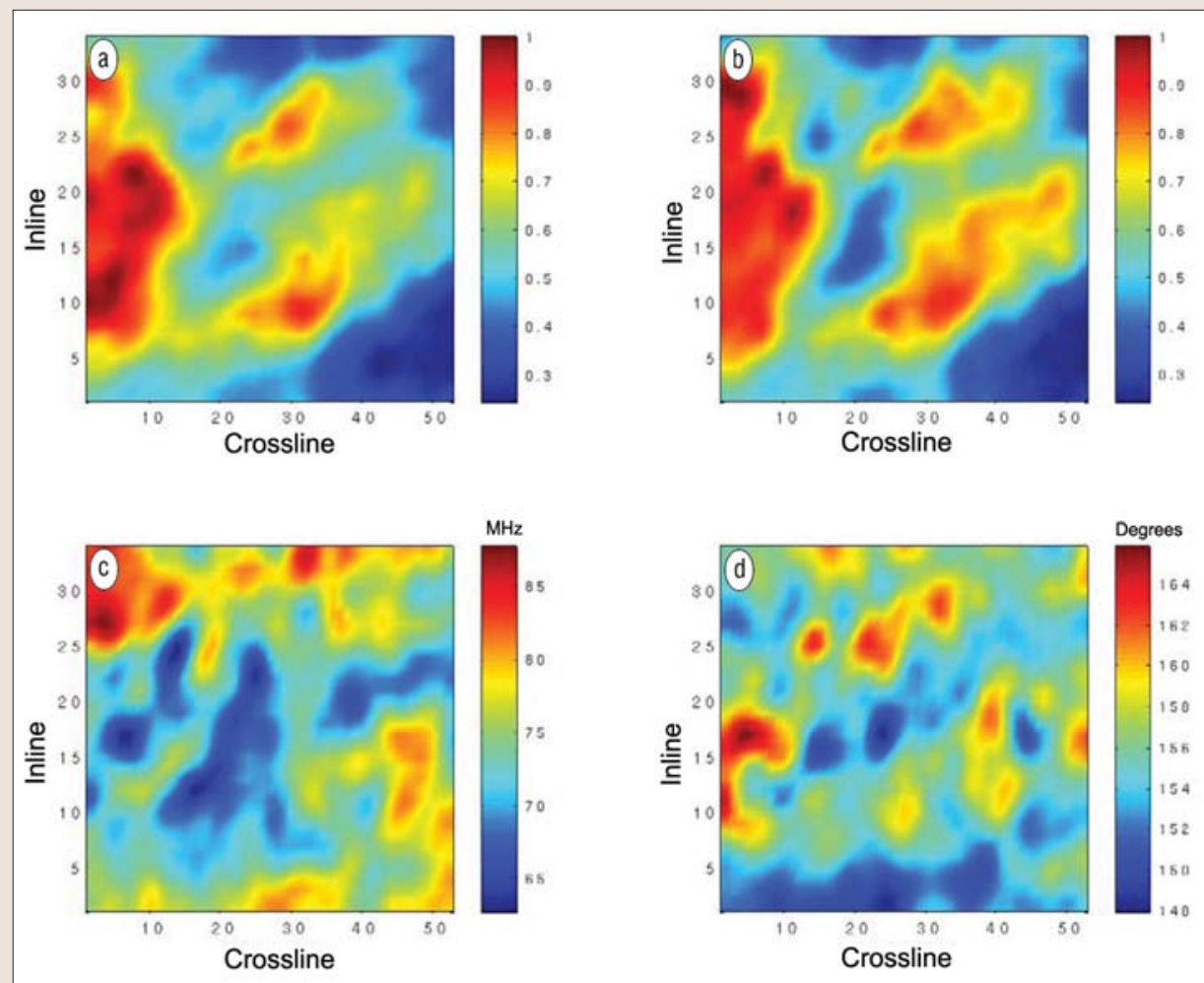

Figure 6. The summed trace (a), instantaneous amplitude or reflection strength (b), and instantaneous frequency $(c)$ attributes show down-gradient channeling with a prominent feature oriented in the inline direction with axis at approximately crossline 20. The instantaneous phase (d) shows relatively little variation and does not appear to be sensitive to the channeling.
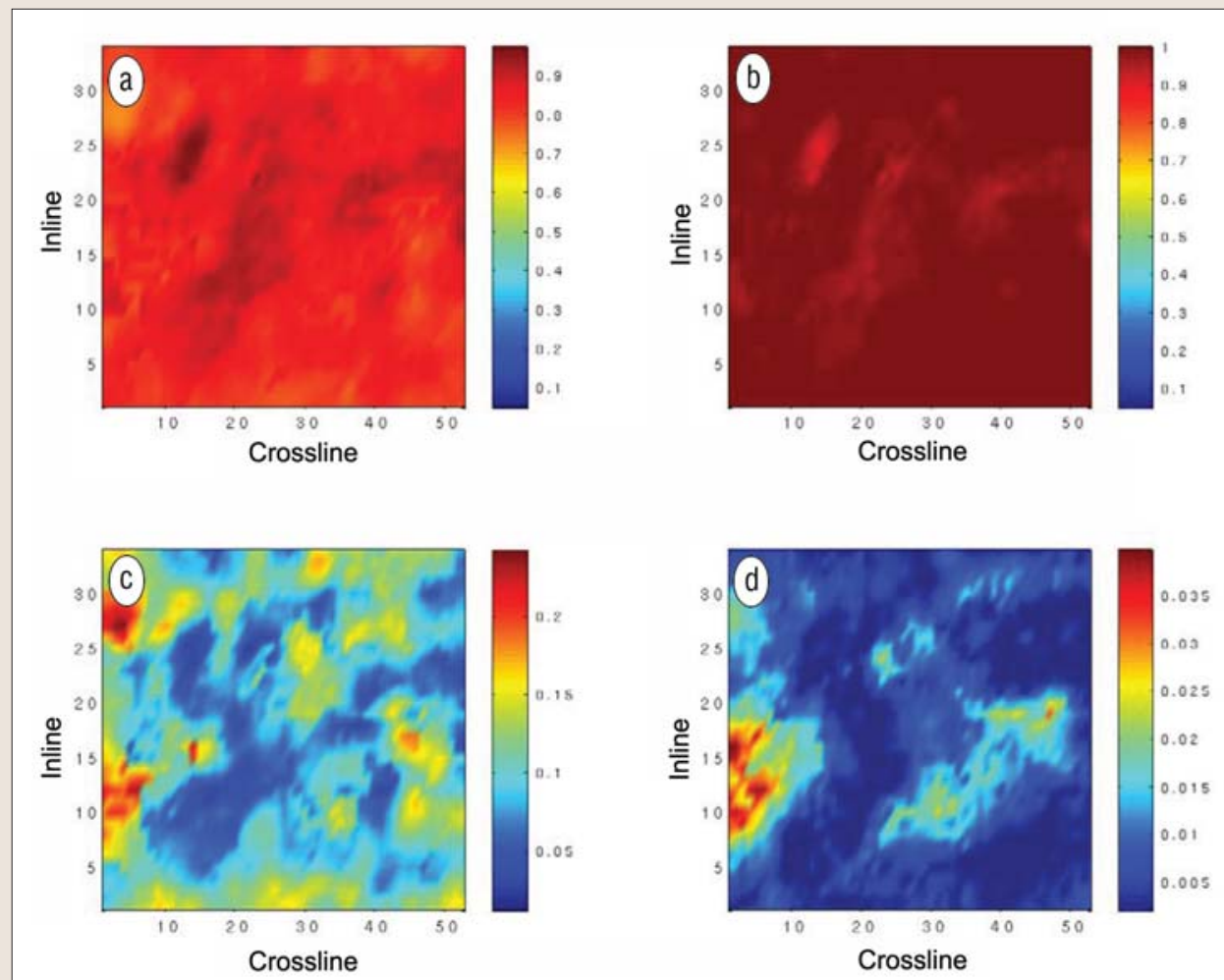

Figure 7. Relative spectral amplitude values along slices through the water table horizon at (a) 47.5 $\mathrm{MHz}$, (b) $67.5 \mathrm{MHz}$, (c) $147.5 \mathrm{MHz}$, and (d) $222.5 \mathrm{MHz}$. The 222.5-MHz slice shows a clearly defined channel feature meandering through the $3 D$ volume. This feature is enriched in low frequencies and depleted in high frequencies, although at the dominant frequency $(\sim 67.5 \mathrm{MHz})$, amplitude varies little throughout the volume. The channel feature appears to correlate with the leftward boundary of the residual phase NAPL. Spectral decomposition results in a significant increase in information content in the attribute images. is directly proportional to $|\mathrm{WMD}|^{2}$ at $t=t_{i}$ (Bradford, 1999).

If we consider a multicomponent signal, we find that the WD of the complex trace produces strong cross-term interference which also makes this distribution undesirable (Bradford, 1999). The STFT does not suffer from cross-term interference, but has poor time resolution properties (Figure 2). Our distribution, WMD, has good time localization properties, and no cross-term interference. Additionally, | WMD | is locally proportional to the amplitude spectra of the zero-phase wavelet and $|\mathrm{WMD}|^{2}$ is locally proportional to the power spectra.

Field example. The field site is a former fire training facility, designated FT-02, located on the now decommissioned Wurtsmith AFB, in Oscoda, Michigan (Figure 3). Over a period of about 24 years, large quantities of fuel were burned on open ground during weekly training exercises. A significant volume of hydrocarbons did not burn and seeped into the underlying aquifer. In 1982, a concrete catch basin was constructed to minimize the amount of contaminant reaching the subsurface. By the early 1990s, the free product plume was up to $0.3 \mathrm{~m}$ thick, and extended more than $200 \mathrm{~m}$ downgradient from FT-02 (Bermejo et al., 1997; Sauck et al., 1998).

The stratigraphy below the site consists of fine-to-medium grained sand and gravel deposits extending to a depth of approximately $20 \mathrm{~m}$. Below this is a $6-30 \mathrm{~m}$ thick silty clay layer which is thought to be the lower boundary for contaminant migration. The surficial aquifer is unconfined, with the water table 3-5 m below the surface. The site was formerly a National Environmental Technology Test Site (NETTS), and was the site of a long-term natural bioremediation investigation. Although no longer an active NETTS site, the wealth of characterization data available make this an excellent location for a semicontrolled GPR field experiment. More recently a soil vapor extraction system was installed, 


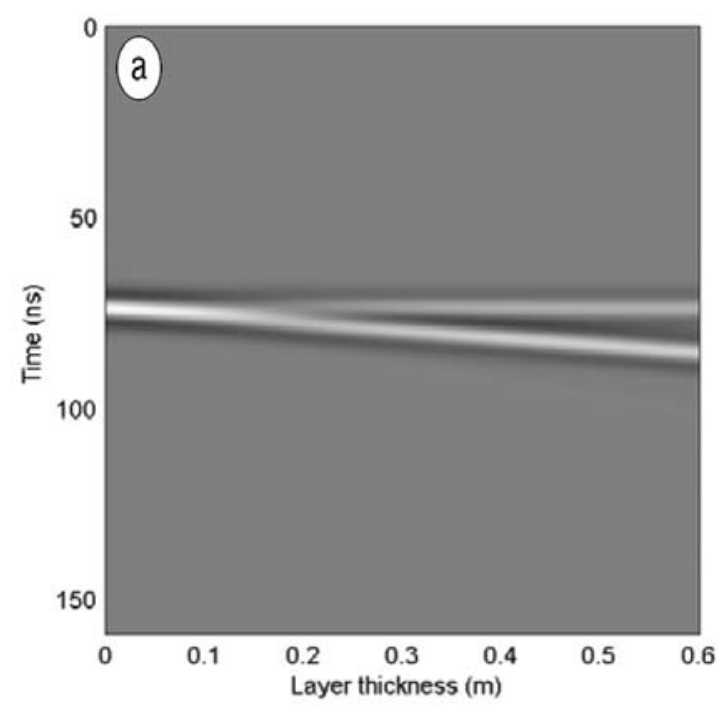

we selected the data set as a good candidate to test whether time-frequency spectral mapping could identify LNAPLrelated anomalies.

The 3D data set consists of parallel, multioffset, $100 \mathrm{MHz}$ GPR profiles covering a $30 \times 30 \mathrm{~m}$ area. Detailed acquisition parameters are given in Table 1. Data processing included to correction, bandpass filtering, a $t^{2}$ gain correction, NMO velocity analysis, and stacking. Additionally, we used the reflection tomography method of Stork (1992) to compute an accurate depth velocity model. NMO stacked profiles have high signal-to-noise with the contaminated interval clearly indicated by a zone of increased attenuation (Figure 4). The attenuated region is collocated with anomalously high velocities just below the water table at about $5 \mathrm{~m}$ depth (Figure 4). The velocity increase is consistent with a maximum volumetric LNAPL concentration of $\sim 10 \%$ using the CRIM equation and assuming constant porosity. We interpret the velocity anomaly as the zone of residual contamination associated with vertical smearing of the contaminant during seasonal fluctuation of the water table.

The 3D stacked data volume shows reflections evident to greater than $400 \mathrm{~ns}$ with the prominent water table horizon, at about 60 ns, continuous throughout the volume (Figure 5). Based on earlier characterization work, we expect an approximately $0.3 \mathrm{~m}$ thick zone of residual LNAPL just above the water table. This thickness is below the conventional image resolution at $100 \mathrm{MHz}$, but the tuning response may be imaged through attribute analysis and is the focus of this study. Prior to spectral decomposition and attribute computation, we applied a 5 trace $\times 5$ trace 3D triangular smoother.

For comparison, we plot the summed trace amplitude (SA), instantaneous amplitude (IA), instantaneous frequency (IF), and instantaneous phase (IPH) along slices through the water-table horizon (Figure 6). Note that the mapped plume area extends from approximately crossline 25 to the right most extent of the 3D area (Figure 3). The SA, IA, and IF distributions show low values along an apparent downgradient channel with an axis located roughly along crossline 20. Based on the earlier work of Bradford (2006), we interpret the lows as reflectivity anomalies associated with the thin layer $(0.3 \mathrm{~m})$ of residual LNAPL just above the water table. The SA is strongly influenced by the water table reflection amplitude since this event dominates the trace amplitude but further indicates decreased trace energy below the water table due to strong attenuation within the plume. The variability in the attributes suggests significant heterogeneity in the contaminant distribution at the water table, and good correlation of the spatial distribution of attributes provides confidence that the anomalies are not merely noise. The IPH shows relatively small variability and no coherent features that are consistent with the geometry of the other three attributes suggesting that the IPH response is relatively insensitive to the contaminants in this case. Downgradient channeling is consistent with the amplitude (A) x gradient (B), amplitude versus offset attribute mapped by Bradford (2003) and was shown by Bradford and Deeds (2006) to be consistent with thin layer of LNAPL at or just above the water table.

Figure 7 shows slices along the water table at $47.5 \mathrm{MHz}$, $67.5 \mathrm{MHz}$ (roughly the average dominant frequency of the water table reflection), $147.5 \mathrm{MHz}$, and $222.5 \mathrm{MHz}$. The plots are scaled so that the relative color range for each plot is identical. It is immediately apparent that the reflection energy in the channel zone is enriched in low frequencies and depleted in high frequencies. It is also interesting to note 
that variation at the dominant frequency is relatively small (see 67.5 MHz plot). At higher frequencies the channel feature becomes increasingly apparent and is well defined at $222.5 \mathrm{MHz}$. The amplitude of the spectrum at $222.5 \mathrm{Mhz}$ varies by a factor of $\sim 10$ whereas the instantaneous frequency varies by a factor of $\sim 1.5$. So, in addition to providing much better lateral resolution, the high-frequency component of the spectrum is a much more sensitive indicator of the channel feature. We computed the reflection response to the thin layer using the reflectivity method and the permittivity model estimated by Bradford and Deeds (2006) to evaluate the spectral response at the water table (Figure 8). The instantaneous spectrum above $200 \mathrm{MHz}$ shows a sharp minima at a layer thickness of around $10 \mathrm{~cm}$, while the spectra at lower frequencies shows a gradual decrease in amplitude. We conclude that the spectral decomposition has substantially increased the information content in our attribute images. The channel feature imaged with attributes is offset from the contaminant plume interpreted based on bulk attenuation of the signal (Figures 3 and 4). Two endmember interpretations are that either the residual LNAPL is not coincident with the dissolved phase plume or that the attributes are imaging stratigraphic features unrelated to the contaminants. Recognizing that small-scale stratigraphy and contaminant transport are inherently linked, it is more likely that the attribute response is indicative of a combination of fluid distribution and geology.

Conclusions. The wavelet matching spectral decomposition is an efficient approach to computing the time-frequency distribution that has good spectral and time resolution and is therefore well suited to GPR imaging problems. Spectral decomposition has proved useful in seismic exploration. We have shown that it also holds significant potential for GPR imaging and, in particular, for detection of groundwater contaminants. It is clear from the spectral plot that variations in the thickness of a thin-bed can cause significant variability in the spectral amplitude that is a function of frequency. Minima or maxima at a specific frequency may be a reliable indicator of bed thickness in the case of a simple three-layer model. However, significantly different bed thicknesses can also produce the same spectral amplitude so as with all geophysical measurements there is clearly nonuniqueness in the measurement. There are of course many other potential applications where imaging below the conventional resolution of the signal holds significant value.

Suggested reading. "Investigations of geoelectrical signatures at a hydrocarbon contaminated site" by Atekwana et al. (Journal of Applied Geophysics, 2000). "Geophysical discovery of a new LNAPL plume at the former Wurtsmith AFB, Oscoda, Michigan" by Bermejo et al. (Groundwater Monitoring and Remediation, 1997). "Time-varying signal processing using the WignerDistribution time-frequency signal representation, Advances in geophysical data processing" by Boudreaux-Bartels (JAI Press Inc. 2, 1985). "Characterizing shallow aquifers with wave-propagation based geophysical techniques: Imaging and attribute analysis" by Bradford (PhD thesis, Rice University, 1999 ). "GPR offset-dependent reflectivity analysis for characterization of a high conductivity LNAPL plume" by Bradford (SAGEEP 2003 Symposium on the Application of Geophysics to Environmental and Engineering Problems, Environmental and Engineering Geophysical Society). "Frequency dependent attenuation analysis of ground-penetrating radar data" by Bradford (GEOPHYSICS, 2007). "Ground-penetrating radar theory and application of thinbed offset dependent reflectivity" by Bradford and Deeds (GEOPHYSICS, 2006). "Time-frequency representation of seismic signals via matching pursuit decomposition with complex Ricker wavelets" by Bradford and Wu (American Geophysical Union, EOS Supplement, 1997). "Ground-penetrating radar monitoring of a controlled DNAPL release: $200 \mathrm{MHz}$ radar" by Brewster and Annan (GEOPHYSICS, 1994). "Monitoring of a controlled LNAPL spill using ground-penetrating radar" by Campbell et al. (SAGEEP 1995 Symposium on the Application of Geophysics to Environmental and Engineering Problems, Environmental and Engineering Geophysical Society). "Instantaneous spectral analysis: Detection of low-frequency shadows associated with hydrocarbons" by Castagna et al. (TLE, 2003). "Frequency-time decomposition of seismic data using wavelet-based methods" by Chakraborty and Okaya (GEOPHYSICS, 1995). "Seismic attributesA historical perspective" by Chopra and Marfurt (GEOPHYSICS, 2005). Ten Lectures on Wavelets by Daubechies (SIAM, 1991). "Geophysical monitoring of a controlled kerosene spill" by DeRyck et al. (SAGEEP 1993 Symposium on the Application of Geophysics to Environmental and Engineering Problems, Environmental and Engineering Geophysical Society). "Timefrequency decomposition based on Ricker wavelet" by Liu et al. (SEG 2004 Expanded Abstracts). "Matching pursuit with timefrequency dictionaries" by Mallat and Zhang (IEEE Transactions in Signal Processing, 1992). "Properties of light nonaqueous phase liquids and detection using commonly applied shallow sensing geophysical techniques" by Monier-Williams (SAGEEP 1995 Symposium on the Application of Geophysics to Environmental and Engineering Problems, Environmental and Engineering Geophysical Society). "Detection and analysis of LNAPL using the instantaneous amplitude and frequency of ground-penetrating radar data" by Orlando (Geophysical Prospecting, 2002). "Complex seismic trace analysis of thin beds" by Robertson and Nagomi (GEOPHYSICS, 1984). "High conductivities associated with an LNAPL plume imaged by integrated geophysical techniques" by Sauck et al. (Journal of Environmental and Engineering Geophysics, 1998). "Spectral decomposition of seismic data with continuous-wavelet transform" by Sinha et al. (GEOPHYSICS, 2005). "Reflection tomography in the postmigrated domain" by Stork (GEOPHYSICS, 1992). TLE

Corresponding author: johnb@cgiss.boisestate.edu 